\section{SUPERINFECTION WITH STREPTOCOCCUS PNEUMONIAE DURING THERAPY WITH CIPROFLOXACIN}

Ciprofloxacin is a newly released fluoroquinolone antibiotic that has been approved for use in a variety of community-acquired infections, including lower respiratory tract infections. This approval has met with some skepticism, in that the in vitro activity of this agent against Streptococcus pneumoniae, the most frequently isolated organism in community-acquired pneumonia, is less than ideal [1].

We report a patient who developed nosocomial pneumococcal pneumonia immediately after completing a course of intravenous ciprofloxacin and nebulized tobramycin for pneumonia due to Pseudomonas aeruginosa. We suspect that the administration of ciprofloxacin and tobramycin allowed colonization and subsequent infection of the respiratory tract to occur with $S$. pneumoniae.

A 61-year-old man entered the Ann Arbor Veterans Administration Medical Center in May 1989 for treatment of recurrent atrial flutter. His past medical history was remarkable for large cell carcinoma of the lung treated with left upper lobectomy in March 1986 and chronic obstructive pulmonary disease.

The patient had a difficult and prolonged hospital course, complicated by acute cholecystitis, renal failure, respiratory failure requiring long-term ventilator support, and recurrent episodes of sepsis.

Pneumonia due to $P$. aeruginosa developed and initially responded to treatment with tobra- mycin and piperacillin. However, recurrence with a new left lower lobe infiltrate developed, and he required reintubation. Sputum cultures revealed $P$. aeruginosa resistant to all antibiotics tested except ciprofloxacin, tobramycin, and amikacin. The patient was treated with ciprofloxacin, $200 \mathrm{mg}$ intravenously every 12 hours, and nebulized tobramycin, $25 \mathrm{mg}$ every 8 hours, for 14 days with complete clinical and roentgenographic resolution of the pneumonia.

One day after completing this course of therapy, the patient again developed dyspnea, fever, tachycardia, and a new right lower lobe infiltrate. Sputum revealed many neutrophils and gram-positive diplococci on Gram's stain and yielded a pure culture of S. pneumoniae. Blood cultures showed no growth. He was treated with penicillin $G, 1$ million units intravenously every 4 hours, with resolution of his illness.

Ciprofloxacin has excellent activity against most gram-negative bacteria, making its potential for the treatment of nosocomial pneumonias promising [2]. It also appears to be quite active against a number of organisms commonly observed in community-acquired pulmonary infections, such as Mycoplasma pneumoniae, Haemophilis influenzae, Branhamella catarrhalis, and Legionella pneumophila [2]. However, its activity against Streptococcus pyogenes (group A streptococcus) and anaerobes is poor, and its activity against $S$. pneumoniae is only marginal [1].

Studies documenting the efficacy of ciprofloxacin in the therapy of pneumococcal pneumonia are lacking; in fact, failure to respond to ciprofloxacin, with clini- cal worsening and bacteremia, has been reported in a patient with pneumococcal pneumonia [3]. Several studies have evaluated the efficacy of ciprofloxacin in the treatment of chronic bronchitis. Davies et al [4] reported good response rates for patients treated with ciprofloxacin for exacerbations of chronic bronchitis, unless $S$. pneumoniae was the inciting microbe. Hoogkamp-Korstanje and Klein [5] found that the pneumococcus was slowly eradicated from sputum in patients with exacerbations of chronic bronchitis treated with ciprofloxacin; the organism was still present as long as 1 week after therapy had begun.

Difficulty in eradicating the pneumococcus from sputum has been attributed to the relatively high minimal inhibitory concentrations of ciprofloxacin for this organism, which range from 0.5 $\mu \mathrm{g} / \mathrm{mL}$ to $4 \mu \mathrm{g} / \mathrm{mL}$ [1]. Serum levels of ciprofloxacin during therapy with both intravenous and oral preparations are quite variable. Mean peak serum levels of ciprofloxacin after $200 \mathrm{mg}$ given intravenously range from 3.2 to $6.3 \mu \mathrm{g} / \mathrm{mL}$; however, 1 hour later, levels typically drop to 0.9 to 1.5 $\mu \mathrm{g} / \mathrm{mL}$. With oral therapy, mean peak serum levels are usually no greater than $3 \mu \mathrm{g} / \mathrm{mL}$ [6]. Davies et al [4] reported a mean peak ciprofloxacin level in sputum of only $1.57 \mu \mathrm{g} / \mathrm{mL}$ at a dose of 750 mg twice a day, which was not high enough to inhibit many strains of $S$. pneumoniae. However, others have reported concentrations of ciprofloxacin in pulmonary secretions or lung tissue several times greater than those found in serum $[7,8]$.

The development of nosocomial pneumonia can be viewed as a multistage process, beginning 
with alteration of the host's natural microbial flora, colonization of the upper respiratory tract with potential pathogens, subsequent aspiration of these organisms, and the development of pneumonia. Gram-negative bacilli most commonly colonize the respiratory tract in patients who are seriously ill, especially those requiring ventilator support in the intensive care unit setting [9]. Hence, these organisms account for the vast majority of pathogens in nosocomial pneumonia, as opposed to the pneumococcus, which accounts for approximately $3 \%[10]$.

We hypothesize that our patient developed pneumococcal pneumonia as a result of eradication of gram-negative colonization by the combined effects of ciprofloxacin and nebulized tobramycin. Both agents have outstanding activity against gramnegative pathogens and poor-tomarginal activity against $S$. pneumoniae.

This experience may also be a valuable lesson for the outpatient setting. Ciprofloxacin is being dispensed with great frequency for the therapy of community-acquired lower respiratory tract infections. Treatment failure may occur when the primary pathogen is the pneumococcus; these failures could be avoided with more conventional therapy. We would like to alert physicians to this possibility and discourage the indiscriminate use of ciprofloxacin for respiratory infections when the pneumococcus is believed to be a likely etiologic agent.

JAMES J. GORDON, M.D. CAROL A. KAUFFMAN, M.D. Veterans Administration Medical

Center

University of Michigan Medical School

Ann Arbor, Michigan

1. Kayser FH, Novak J. In vitro activity of ciprofloxa-
cin against gram-positive bacteria. Am J Med I987;
82 (Suppl 4A): $33-9$.
2. Koester JA. Ciprofloxacin: a new fluoroquinolone.
Am J Med Sci 1988; 297: 128-31.

3. Cooper B, Lawlor M. Pneumococcal bacteremia during ciprofloxacin therapy for pneumococcal pneumonia (brief clinical observation). Am J Med 1989; 87: 475.

4. Davies BI, Maesen FPV, Baur C. Ciprofloxacin in the treatment of acute exacerbations of chronic bronchitis. J Antimicrob Chemother 1986; 5: 22631.

5. Hoogkamp-Korstanje JAA, Klein SJ. Ciprofloxacin in acute exacerbations of chronic bronchitis. J Antimicrob Chemother 1986; 18: 407-13.

6. Lode H, Hoffken G, Borner K, Koeppe P. Ciprofloxacin intravenous dose variance. Am J Med 1989: 87 (Suppl 5A): 40-5.

7. Reid TMS, Gould IM, Golder D, et al. Brief report: Respiratory tract penetration of ciprofloxacin. Am J Med 1989; 87 (Suppl 5A): 60-1.

8. Schlenkhoff D, Dalhoff A, Knopf J, Opferkuch W. Penetration of ciprofloxacin into human lung tissue following intravenous injection. Infection 1986; 14: 299-300.

9. Johanson WG, Pierce AK, Sanford JP, Thomas GD. Nosocomial respiratory infections with gramnegative bacilli: the significance of colonization in the respiratory tract. Ann Intern Med 1972; 77 : 701-6.

10. Jay SJ. Nosocomial infections. Med Clin North Am 1983; 67: 1251-77.

Submitted January 12, 1990, and accepted in revised form April 30, 1990

\section{SUCCESSFUL USE OF GRANULOCYTE-MACROPHAGE COLONY-STIMULATING FACTOR IN PATIENTS WITH ACUTE LYMPHOCYTIC LEUKEMIA}

Granulocyte-macrophage colony-stimulating factor (GM-CSF) is a recombinant human hematopoietic factor with the potential to stimulate the growth of neutrophil precursors, as well as activate those already produced. It has been used successfully to prevent or treat the neutropenia associated with the treatment of solid tumor [1]; to stimulate the growth of normal precursors in aplastic anemia [2], hairy cell leukemia [3], and acquired immunodeficiency syndrome [4]; and to reverse some cyclic neutrophil disorders [5]. However, it has also shown the potential to stimulate the growth of disordered clones of hematopoietic cells found in patients with advanced myelo- dysplastic syndrome [6], and some researchers have expressed concern that some solid tumors may have receptors for GM-CSF and be susceptible to its action [7]. Despite this concern, prior studies with malignant B cells in vitro failed to demonstrate any malignant growth potentiation [8]. We describe here the successful use of GM-CSF to treat infections in two patients with acute lymphocytic leukemia (ALL) associated with profound neutropenia secondary to chemotherapy. No stimulation of growth of ALL blasts was evident during or after treatment with GM-CSF.

Patient 1. A 23-year-old man developed pre-auricular adenopathy that was diagnosed as nodular well-differentiated lympho$\mathrm{ma}$, and he was treated with chlorambucil. After a brief response, rapid regrowth of lymph nodes occurred and therapy was switched to oral cyclophosphamide. One month later he presented with fever, chills, splenomegaly, and a white blood cell count of $150,000 / \mathrm{mm}^{3}$, and the diagnosis of ALL was made. The patient began to receive daunorubucin, vincristine, and prednisone, with a rapid decrease in the white blood cell count to $800 /$ $\mathrm{mm}^{3}$. He was transferred to the Medical College of Virginia, where bone marrow biopsy and aspiration confirmed ALL, and cytogenetic study revealed positive Philadelphia chromosome, trisomy 17 , monosomy 8 , and monosomy 14 . Immunophenotyping disclosed that the cells were pre- $B$ cells. The L17-M regimen [9] of cyclophosphamide, vincristine, and prednisone was begun, with a rapid lysis of the peripheral blood blasts. However, bone marrow biopsy showed hypercellularity with $100 \%$ blasts. He was subsequently treated with methotrexate, vincristine, asparaginase, and dexamethasone [10], with rapid lysis 